\title{
Effects of Enteral Immunonutrition in Esophageal Cancer
}

\author{
Kenji Mimatsu ${ }^{a}$ Nobutada Fukino ${ }^{a}$ Yasuo Ogasawara ${ }^{a}$ Yoko Saino ${ }^{b}$ \\ Takatsugu Oidac \\ Departments of a Surgery and ${ }^{b}$ Nutrition, Japan Community Health Care Organization \\ Yokohama Central Hospital, Yokohama, and 'Department of Surgery, Kiba Hospital, Tokyo, \\ Japan
}

\section{Keywords}

Chemoradiotherapy · Esophageal cancer · Immunonutrition · Surgery

\begin{abstract}
Background: Immunonutrition (IN) significantly reduces the incidence of postoperative infectious complications and the length of hospitalization in patients undergoing major elective surgery for gastrointestinal malignances. However, the clinical benefit of IN in patients who have undergone esophagectomy for esophageal cancer is unclear. Moreover, the effect of enteral IN in patients during preoperative adjuvant chemoradiotherapy and in patients treated with concurrent chemoradiotherapy for advanced esophageal cancer is unknown. Summary: This review analyzes the evidence supporting the enteral administration of IN in patients who have undergone esophagectomy and/or chemoradiotherapy for esophageal cancer. Twelve trials that evaluated IN exclusively in patients who underwent esophagectomy were published between January 1980 and August 2017. Two trials concerning IN during chemoradiotherapy for esophageal cancer were identified in the same period. However, the evidence is insufficient to recommend enteral IN in patients who have undergone esophagectomy and/or chemoradiotherapy for esophageal cancer. Key Message: Further evidence from well-designed randomized controlled trials is required to verify the clinical benefits of enteral IN in patients undergoing esophagectomy and/or chemoradiotherapy for esophageal cancer. Practical Implications: Resolvins, which are generated from EPA, are novel anti-inflammatory lipid mediators and may play a key role in the resolution of acute inflammation when IN is supplemented with EPA in patients undergoing severely stressful operations.
\end{abstract}




\section{Introduction}

\section{Incidence of Malnutrition and Nutritional Support}

The majority of patients with esophageal cancer have a poor nutritional status. Esophageal cancer patients show weight loss and an elevated nutritional risk [1]. In the report of a large American series, 57.3\% of esophageal cancer patients presented with weight loss at the time of diagnosis [2]. Van der Schaaf et al. [3] reported that $17 \%$ of patients with esophageal cancer had $>10 \%$ unintentional weight loss during the 3 months prior to diagnosis. This poor nutritional status is considered to be associated with morbidity and mortality after surgery. A multivariable analysis showed that malnutrition and nutritional insufficiency were independent risk factors for postoperative infectious complications and mortality as well as increased the length of hospitalization ( $\mathrm{LOH})$ and hospital costs in cancer patients hospitalized for surgery [4]. The poor nutritional status of patients with esophageal cancer is closely related to postoperative complications [5]. The ESPEN Guidelines recommended the administration of preventive preoperative clinical nutrition support to patients with malnutrition or at risk of malnutrition [6]. Some reports have confirmed that appropriate nutritional support for patients with or at risk of malnutrition can improve the postoperative clinical outcomes, including infectious complications [7, 8]. Thus, although studies on preoperative nutritional support for esophageal cancer patients are lacking, preoperative nutritional support has become an important part of the multidisciplinary treatment of patients undergoing esophagectomy [9].

\section{Immunonutrition in Esophagogastric Cancer}

The administration of sufficient energy and protein is an important method of nutritional management in patients receiving major surgery. In addition, recently, it has been challenging to control the immune system and inflammatory responses using immunonutrition (IN). A number of immunonutrients are available, such as arginine, glutamine, omega-3 polyunsaturated fatty acids ( $\omega-3$ PUFAs), and antioxidants. These substrates have been revealed to upregulate host immune responses and control inflammatory responses, demonstrating their clinical benefit [10]. In particular, immune-enhancing diets (specifically IMPACT ${ }^{\circledR}$ ) have been associated with significantly fewer infectious complications in elective gastrointestinal surgery patients compared with a normal diet [11-13].

Esophagogastric cancer surgery has a higher complication and mortality rate than surgeries for other major malignancies, and the appropriate application of IN may be able to improve patient outcomes after surgery. In the ESPEN Guidelines, the use of immune-modulating formulae enriched with arginine, $\omega$-3 PUFAs, and nucleotides is a grade A recommendation in patients with obvious severe nutritional risk undergoing major cancer surgery of the abdomen, such as esophagectomy, gastrectomy, and pancreatoduodenectomy [14]. In addition, a meta-analysis found that enteral IN significantly reduces the incidence of postoperative infectious complication and the $\mathrm{LOH}$ in patients undergoing major elective surgery for gastrointestinal malignances [12]. To date, a systematic review and meta-analysis by Wong and Aly [15] has demonstrated that enteral IN decreases wound infection rates and reduces $\mathrm{LOH}$, and that it should be recommended as routine nutritional supportas part of the Enhanced Recovery after Surgery (ERAS ${ }^{\circledR}$ ) programs for upper gastrointestinal surgery.

However, thus far, trials have been conducted in heterogeneous patient groups [16], hampering the establishment of recommendations for specific patient groups. Although a few trials have included patients undergoing esophageal cancer resection [17-19], these patients generally comprised only a small subset of the total study population, and thus those trials are inadequate to prove the effectiveness of IN in patients with esophageal cancer. Moreover, the effects of enteral IN in patients during preoperative adjuvant chemoradiotherapy and in 
patients treated with concurrent chemoradiotherapy for advanced esophageal cancer are unknown. Thus, this review was conducted to assess the available evidence on the clinical benefit of IN alone in patients with esophageal cancer undergoing esophagectomy and/or chemoradiotherapy.

\section{Methods}

Retrieval of studies was performed through MEDLINE (via PubMed), the Cochrane Library databases, and Google Scholar for randomized controlled trials (RCTs), meta-analyses, or retrospective studies to determine the clinical benefits of IN in patients undergoing esophagectomy and/or chemoradiotherapy for esophageal cancer. Medical subject heading (MeSH) terms were used to search electric databases. The terms "esophageal cancer" OR "esophagectomy" OR "surgery" OR "resection" OR "operative" OR "immunonutrition" OR "immune-enhancing" OR "immune-enhanced" OR "immune-modulating” OR "chemotherapy" OR "radiotherapy" OR "chemoradiotherapy" were used as the selected keywords. This search was based on the combination of publication type "Clinical trials or randomized controlled trials or meta-analysis" and publication date between January 1980 and August 2017. We also conducted a cross-reference check and a citation search. Clinical trials published as full papers with an abstract in English in peer-reviewed journals were selected. In this study, an IN diet included at least one of the following nutrients: arginine, glutamine, $\omega-3$ PUFAs, or antioxidants.

Eligible journals were analyzed based on the following: (1) type of study, (2) evidence level according to classification advocated by the Agency for Health Care Policy and Research, and (3) outcome measurements (postoperative complications, including infectious and noninfectious complications and $\mathrm{LOH}$ ).

\section{Results}

\section{IN in Patients Undergoing Esophagectomy}

Evidence Level and Study Design. Twelve trials [17-28] that evaluated IN exclusively in esophageal cancer patients who underwent esophageal resection were published between January 1980 and August 2017 (Table 1). The numbers of trials classified by evidence level are as follows: $1 \mathrm{a}, n=0 ; 1 \mathrm{~b}$ (RCT), $n=7 ; 2 \mathrm{a}, n=5 ; 2 \mathrm{~b}, n=0 ; 3, n=0 ; 4, n=0$. Nine of the trials were performed in Japan and three in Europe.

Comparison between Preoperative IN and Control Diet. Four trials [21,23, 25, 27] compared the preoperative administration of IN with an ordinary diet. Three trials were retrospective studies and one was a prospective randomized study. A retrospective audit of a large number of patients ( $n=123$ ) conducted by Fukuda et al. [21] showed that preoperative IN (IMPACT [Ajinomoto Pharma, Tokyo, Japan] or ANOM $^{\circledR}$ [Otsuka Pharmaceutical Co., Tokushima, Japan]) significantly reduced the incidence of catheter-related fever (7 vs. 23\%; $p=0.012$ ) and pneumonia ( $6 \mathrm{vs.} 18 \% ; p=0.037$ ) as well as the postoperative hospital stay ( $27 \pm 1.8 \mathrm{vs}$. $39 \pm 3.7$ days; $p<0.01$ ) compared with the control group (nonsupplemented ordinary diet). Kubota et al.'s study [25] showed that preoperative IN (IMPACT) significantly reduced infectious complication and $\mathrm{LOH}$. Moreover, that trial showed a significant difference in the 6 -month survival rates between the intervention group and the control group (92 vs. $72 \%$; $p=0.028$ ). One prospective randomized trial by Kitagawa et al. [27] evaluated the effects of preoperative IN in patients undergoing thoracoscopic esophagectomy. In that study, MHN-02 (MEIN $^{\circledR}$; Meiji Dairies Co., Tokyo, Japan) as an immune-modulating diet was administrated during the 5 days before surgery. MHN- 02 contains palatinose as a carbohydrate source, ribonucleic acid, whey-hydrolyzed protein, amino acids, enriched $\omega$-3 PUFAs (EPA and DHA), and antioxidants such as vitamins $\mathrm{C}$ and $\mathrm{E}$. Preoperative $\mathrm{MHN}-02$ suppressed the elevation of tumor necrosis factor- $\alpha$ levels, although no different tendency was detected in terms of inter- 
Gastro

Intestinal

Tumors

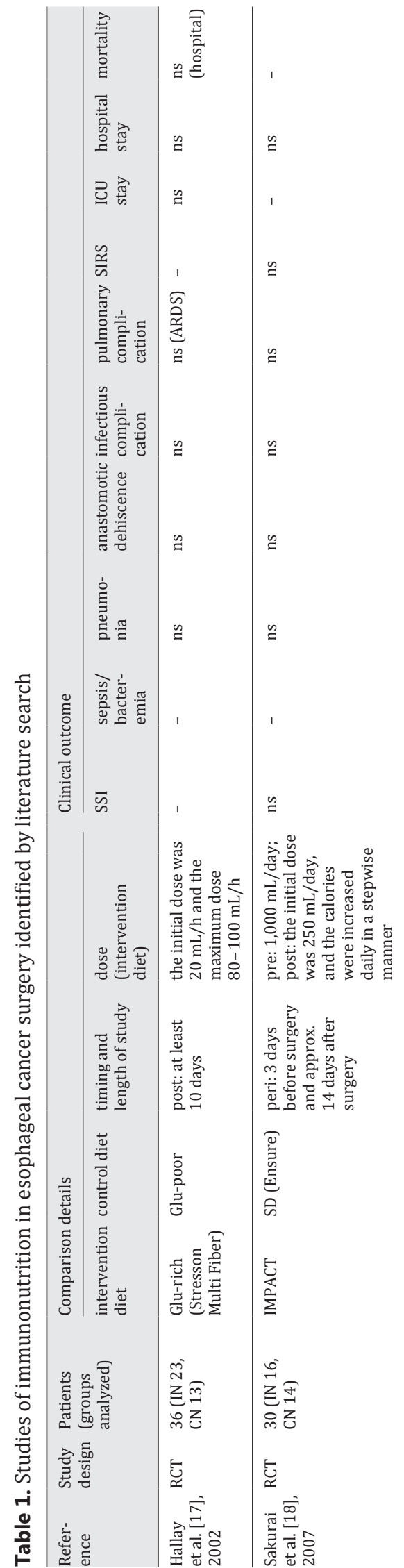

Gastrointest Tumors 2017;4:61-71

Mimatsu et al: Effects of Enteral Immunonutrition in Esophageal Cancer

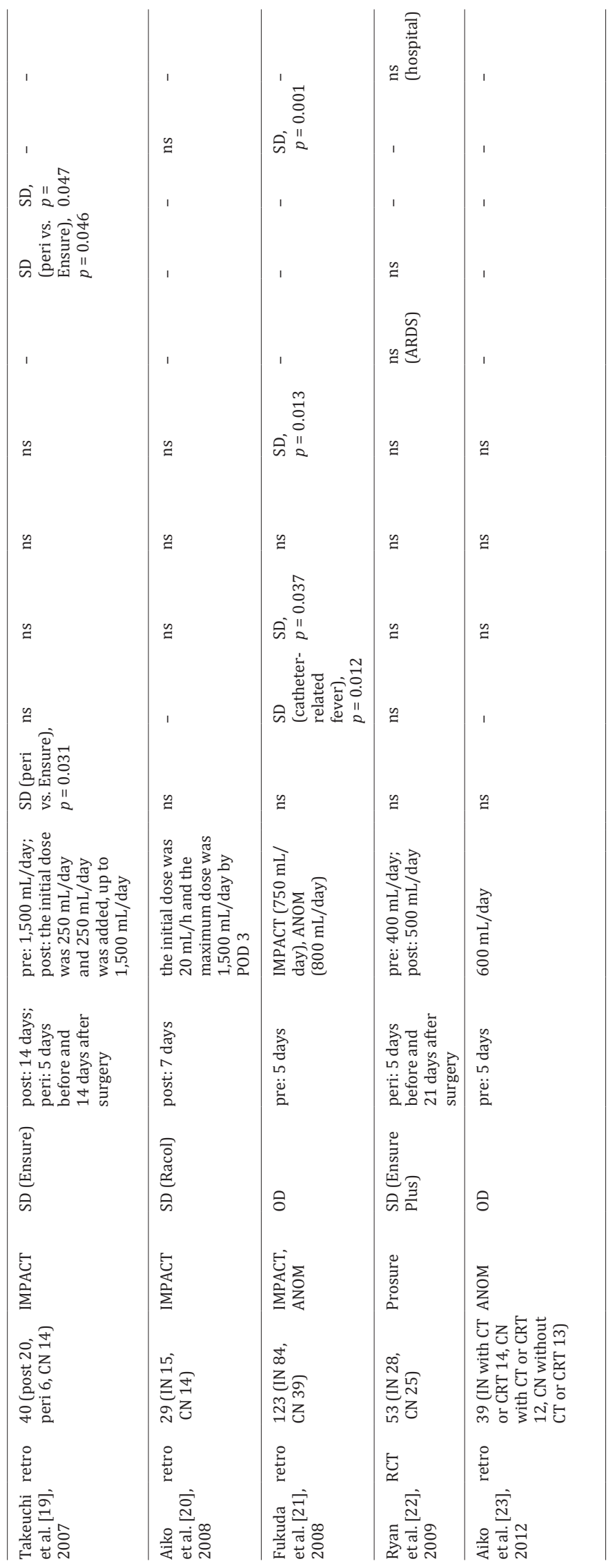


Gastro

Intestinal

Tumors

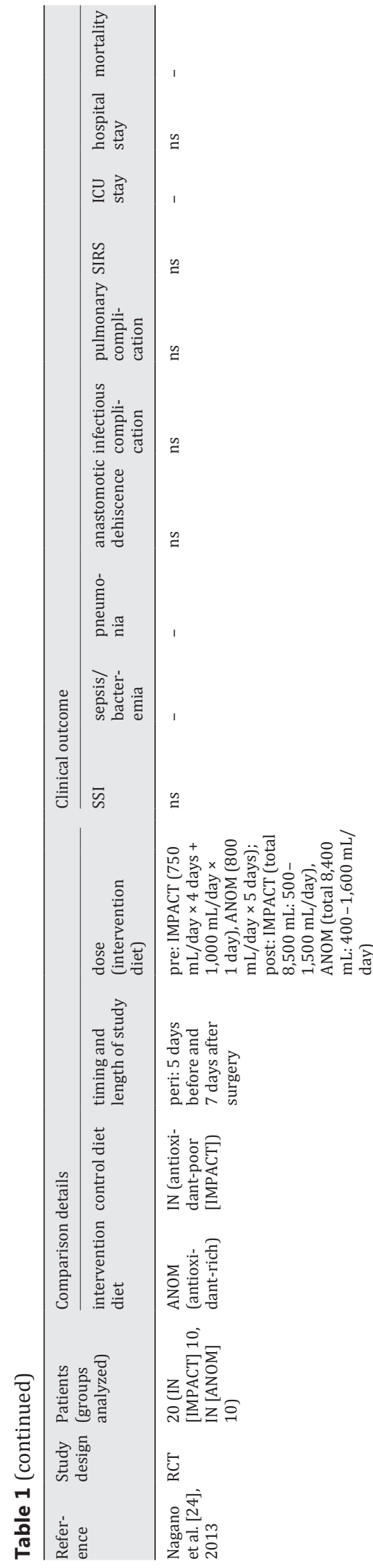

Gastrointest Tumors 2017;4:61-71

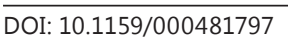

(C) 2017 S. Karger AG, Basel

www.karger.com/gat

Mimatsu et al.: Effects of Enteral Immunonutrition in Esophageal Cancer

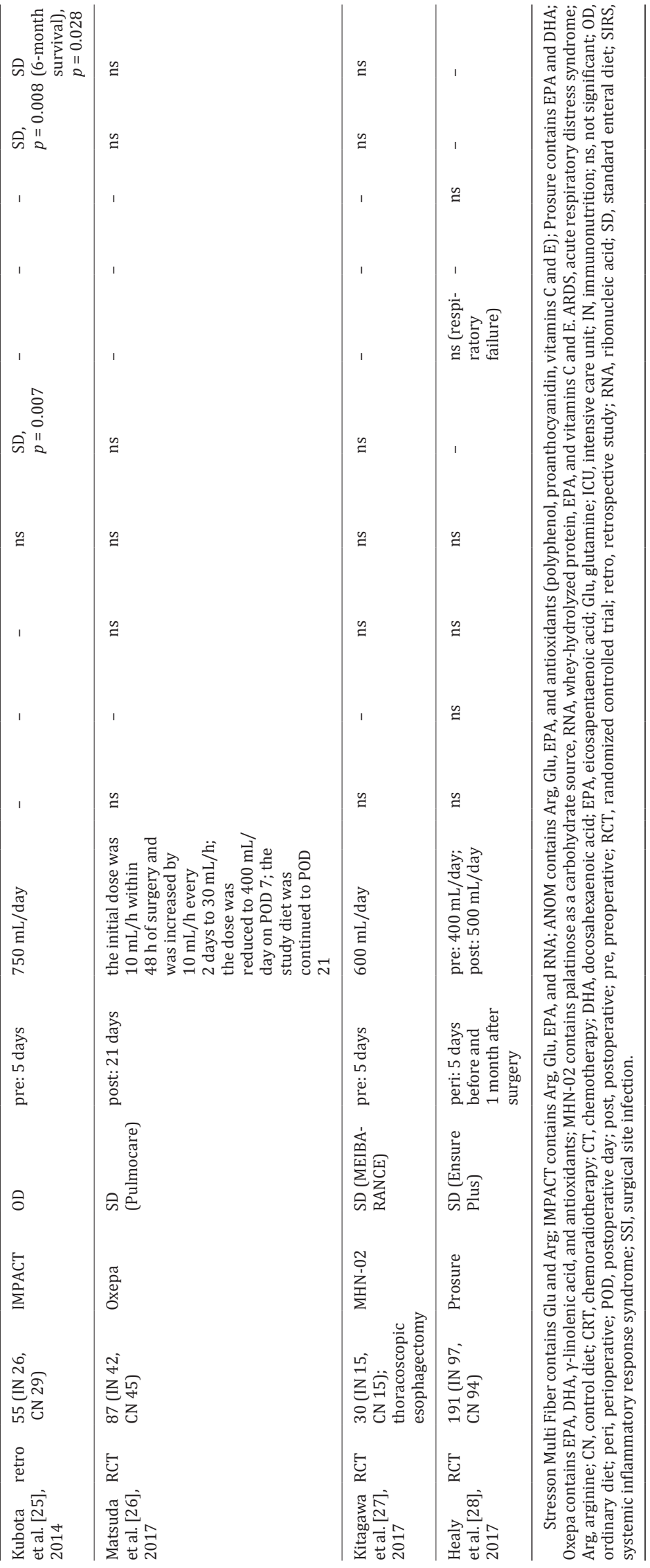


leukin 6 (IL-6), C-reactive protein, or postoperative complications including pneumonia, anastomotic leakage, and surgical site infection (SSI).

IN in Patients Undergoing Preoperative Adjuvant Chemoradiotherapy. The study by Aiko et al. [23] demonstrated whether preoperative IN (ANOM) reduces the incidence of morbidity in patients treated with or without neoadjuvant chemotherapy and/or radiotherapy followed by esophagectomy. ANOM contains arginine, glutamine, EPA, and antioxidants such as polyphenol, vitamin C, and vitamin E. Preoperative administration of ANOM suggested reduction of oxidative stress. However, there was no significant difference in the incidence of complications after surgery between preoperative ANOM and an ordinary diet in patients treated with or without neoadjuvant anticancer therapy followed by esophagectomy.

Comparison between Postoperative IN and a Control Diet. Three trials [17,20,26] compared the postoperative administration of IN with a control diet. Two of these trials were RCTs and one was a retrospective study. Each trial used different nutrients as the intervention target. Moreover, the period of postoperative administration differed in each of the trials. The effect of glutamine-rich enteral nutrition was compared to that of glutamine-poor enteral nutrition in one study [17]. The study showed that there were no significant differences in pulmonary complications, anastomotic dehiscence, or mortality. Aiko et al. [20] reported a retrospective study that compared IN (IMPACT) to standard nutrition (Racol ${ }^{\circledR}$; Otsuka Pharmaceutical Co., Tokyo, Japan). They suggested that early enteral nutrition with IN may enhance the inhibitory effects against postoperative platelet aggregation and hypercoagulation and appeared to improve $\mathrm{T}$ cell proliferation. However, there were no significant differences in the clinical benefits, including SSI, pneumonia, anastomotic dehiscence, and LOH. Recently, Matsuda et al. [26] reported the results of an RCT comparing enteral IN (Oxepa ${ }^{\circledR}$; Abbott Japan, Tokyo, Japan) to a control diet (Pulmocare ${ }^{\circledR}$; Abbott Japan) in patients undergoing radical esophagectomy for thoracic esophageal cancer. The primary outcome measure was the change in the oxygen status $\left(\mathrm{PaO}_{2} / \mathrm{FiO}_{2}\right.$ ratio), and the secondary outcome measures were body composition, inflammation-related factors, coagulation markers, cholesterol concentrations, and clinical outcomes. That trial revealed that oxygenation in the IN group was significantly improved on postoperative days 4, 6, and 8 in comparison to the standard diet group, and that the changes in body weight (on postoperative days 14 and 21) and lean body mass (on postoperative day 21) of the IN group were significantly greater in comparison to the standard diet group. However, no significant differences were observed in clinical outcomes, including the rates of pneumonia, anastomotic leakage, SSI, and in-hospital mortality, or in postoperative $\mathrm{LOH}$.

Comparison between Perioperative IN and Control Diet. Three RCTs [18, 22, 28] compared the perioperative administration of IN with a standard enteral diet. In the first, Sakurai et al. [18] reported an RCT that compared IMPACT to a standard enteral diet (Ensure ${ }^{\circledR}$; Abbott Japan). That trial suggested that perioperative IN was beneficial for maintaining humoral immunity rather than cellular immunity. However, there were no significant difference in the clinical benefit, including the rate of postoperative infectious complications, the duration of systemic inflammatory response syndrome (SIRS), and LOH. The study was limited by the small number of patients and the absence of any blinding. The second study, a doubleblind RCT by Ryan et al. [22], found that perioperative use of Prosure ${ }^{\circledR}$ (Abbott Laboratories, Ireland) containing EPA $2.2 \mathrm{~g}$ /day until 3 weeks after surgery maintained postoperative fat-free mass in patients undergoing esophageal cancer surgery, whereas patients receiving the control isocaloric and isonitrogenous formula (Ensure Plus ${ }^{\circledR}$; Abbott Laboratories, Ireland) lost a significant amount of lean body mass (fat-free mass decreased by $1.9 \mathrm{~kg}, p=0.03)$. However, no significant difference was seen in the length of SIRS or in the rates of SSI, pneumonia, anastomotic dehiscence, or in-hospital mortality. Recently, a multicenter double-blind RCT was reported by Healy et al. [28] in the same group as in Ryan et 
al. [22]. That trial focused not only on short-term outcomes, but also on observations of patients for up to 6 months after surgery. Prosure or Ensure Plus was administered preoperatively 5 days orally and postoperatively via jejunostomy until 1 month after discharge. Unfortunately, administration of EPA failed to suppress the loss of lean body mass, and no significant difference was observed in clinical, nutritional, functional, and quality of life outcomes at the 1,3, and 6 months postoperatively. Moreover, there were no significant difference in postoperative complications (anastomotic leakage, respiratory failure, pneumonia, and sepsis) and the length of intensive care unit stay. A small retrospective study conducted by Takeuchi et al. [19] evaluated three arms: postoperative IN, perioperative IN, and standard enteral diet. That study showed that perioperative IN (IMPACT) significantly reduced the incidence of SSI and the duration of SIRS compared with the standard enteral diet. Nagano et al. [24] reported a pilot study to compare the effects of two types of IN, an antioxidant-enriched enteral nutrition (ANOM) and an antioxidants-poor enteral nutrition (IMPACT) after esophagectomy for esophageal cancer. The results of that study suggested that ANOM and IMPACT have a similar effect on immunoinflammatory response, antioxidant capacity, and clinical outcomes including infectious complications, duration of SIRS, $\mathrm{LOH}$, and body composition.

\section{Safety of IN in Surgery Patients}

There is controversy surrounding the administration of immune-enhancing diets containing arginine to critically ill patients, as high arginine content drives nitric oxide (NO) assembly $[29,30]$. Arginine is a substrate for NO, and NO has vasodilatory action. The supply of NO improves tissue circulation and promotes recovery of local immunity and improvement of tissue damage. However, a large amount of NO produced by macrophages is involved in vasodilatation and hypotension and promotes shock in critically ill patients with sepsis [31] and pneumonia [32]. During the postoperative period, surgical patients go through contrasting stages: SIRS and compensatory anti-inflammatory response syndrome (CARS), which hamper the maintenance of homeostasis. Resection of esophageal cancer is one of the most invasive gastrointestinal surgeries and causes the SIRS and CARS. Arginine may be likely to promote SIRS and CARS. According to the ASPEN guideline [33], the use of immune-enhancing diets containing arginine should be restricted to select groups of critically ill patients, including those undergoing major elective surgery. Although the use of immunonutrients such as glutamine, fish oil, and ribonucleic acid are thought to be less harmful, it is a safety concern to add arginine to the specialized formulae in major surgical patients such as those undergoing esophagectomy. We need to evaluate the safety of the administration of immune-enhancing diets containing arginine in esophageal cancer surgery.

\section{IN in Patients Undergoing Chemoradiotherapy}

Two studies [34, 35] concerning enteral IN during chemoradiotherapy for esophageal cancer were identified. One study by Yoshida et al. [34] examined whether glutamine supplementation would maintain or enhance systemic lymphocyte function and protect gut barrier in patients receiving neoadjuvant chemoradiotherapy for advanced esophageal cancer. Glutamine (Glumine ${ }^{\circledR}$; Kyowa Hakkoh, Tokyo, Japan) was administered orally at $30 \mathrm{~g} /$ day at the start of chemoradiotherapy and for the subsequent 28 days. All patients underwent mediastinal irradiation and chemotherapy consisting of 5-fluorouracil and cisplatin. That study revealed that oral glutamine supplementation protects lymphocytes and attenuates gut permeability in patients with advanced esophageal cancer during chemoradiotherapy. The other study by Sunpaweravong et al. [35] examined whether enteral IN during concurrent chemoradiotherapy reduces inflammation and improves 
immune function in patients with squamous cell carcinoma. The IN group received a combination of EPA, glutamine, and arginine (Neomune ${ }^{\circledR}$; Thai Otsuka Pharmaceutical Co., Thailand), whereas the control group received a standard formula (Modified Blendera ${ }^{\circledR}$; Thai Otsuka Pharmaceutical Co.). That study revealed that enteral IN during concurrent chemoradiotherapy reduced the increase of inflammatory cytokine levels more than the standard formula. However, the clinical side effects to chemoradiotherapy in terms of esophagitis, mucositis, diarrhea, nausea, and vomiting were not significantly different between the treated and the control group.

\section{The Effect for EPA on Esophageal Carcinogenesis}

Many immunonutrients, including $\omega-3$ PUFAs, glutamine, arginine, and antioxidants, have been studied in vitro or in vivo. However, few of these nutrients have shown clinical efficacy when administered individually. EPA, which is a component of $\omega-3$ PUFAs, plays a role in the inhibition of cell proliferation and the anticancer effect. Two studies [36, 37] demonstrated the effect of EPA on an esophageal cancer cell line. One study by Kubota et al. [36] revealed that EPA inhibits NF- $\kappa$ B activation and IL-6 production in esophageal cancer cells, resulting in inhibition of cancer cell proliferation by induction of apoptosis. The other study by Mizoguchi et al. [37] revealed the induction of apoptosis by EPA in esophageal squamous cell carcinoma. These effects of EPA may be beneficial in improving the outcome of cancer surgery and potential as a new treatment for esophageal cancer. An epidemiologic study by Chen et al. [38] implicates a reduced risk of esophageal adenocarcinoma in a population with high fish oil consumption. One possible explanation is the suppression of eicosanoid production through inhibition of cyclooxygenase-2 (COX-2). An experimental study [39] suggested that upregulation of COX-2 is shown to occur in both Barrett's esophagus and esophageal adenocarcinoma. Mehta et al. [40] revealed that an $\omega-3$ fatty acid supplement consisting of $1.5 \mathrm{~g} /$ day of unesterified EPA provided in 500-mg capsules changed $\omega-3$ fatty acid concentrations and reduced COX-2 concentrations in Barrett's esophagus tissue, and thereby inhibited either the development or the progression of Barrett's esophagus.

\section{Conclusions and Direction of Future Research}

The current study identified less well-designed prospective RCTs for evaluating the key clinical benefits in patients undergoing esophagectomy and/or chemoradiotherapy for esophageal cancer. Therefore, the conclusive determination of the clinical benefits of IN for esophageal cancer will require the performance of well-designed prospective RCTs comparing the outcomes between those receiving IN versus an isocaloric isonitrogenous standard diet. In addition, we need to clarify whether pre-, post-, or perioperative IN imbues the greatest clinical benefits. A meta-analysis that highlighted the importance of timing as a clinical consideration in the provision of IN in elective gastrointestinal surgical patients [41] demonstrated statistically significant reductions in infectious complications and $\mathrm{LOH}$ with peri- and postoperative administration, but no differences were seen in postoperative mortality with the provision of IN, irrespective of the timing of administration. The optimal timing of IN administration must also be evaluated in patients undergoing esophageal cancer surgery. Many of the previous trials did not separately evaluate the responses of malnourished and well-nourished patients. A recent RCT in patients undergoing gastrectomy for gastric cancer found no significant difference in the rates of postoperative morbidity or infectious complications, and concluded that routine preoperative use of IN in well-nourished patients undergoing gastric cancer resections was not be recommended [42]. Esophageal cancer patients often have poor 
nutritional status [1-3]. Therefore, patients should be separately evaluated based on their nutritional status in future studies.

In 2000, Serhan et al. [43] identified a novel anti-inflammatory lipid mediator originated from $\omega$-3 PUFAs, which was later named "resolvin." Resolvins generated from EPA and DHA, which are components of $\omega-3$ PUFAs, are termed resolvin $E$ (RvE) and resolvin D (RvD) series, respectively, both act as pro-resolving lipid mediators in inflammation [44]. Recently, one clinical study by Uno et al. [45] investigated the effects of RvE1 in patients undergoing major hepatobiliary resection who received EPA-enriched IN. In that study, preoperative serum EPA levels showed a significant positive correlation with plasma RvE1 levels immediately after surgery, and plasma RvE1 levels showed a negative correlation with plasma IL-6 levels immediately after surgery. Therefore, it is suggested that RvE1 may play a key role in the resolution of acute inflammation when IN is supplemented with EPA in patients undergoing severely stressful operations. These studies may shed light on new therapeutic prospects for EPA in patients with uncontrolled inflammatory responses [26]. These anti-inflammatory lipid mediators may play an important role in the treatment in patients suffering from inflammatory responses after surgery for esophageal cancer.

In conclusion, there is insufficient evidence to recommend enteral IN in patients undergoing esophagectomy and/or chemoradiotherapy for esophageal cancer. Therefore, more well-designed RCTs comparing the outcomes between esophageal cancer patients receiving IN versus a standard diet are needed.

\section{Disclosure Statement}

None of the authors have any sponsorship or funding arrangements relating to their research, and all authors declare no conflicts of interest associated with this paper.

\section{References}

1 Bozzetti F: Screening the nutritional status in oncology: a preliminary report on 1,000 outpatients. Support Care Cancer 2009;17:279-284.

2 Daly JM, Fry WA, Little AG, et al: Esophageal cancer: results of an American College of Surgeons Patient Care Evaluation Study. J Am Coll Surg 2000;190:562-572; discussion 572-573.

3 Van der Schaaf MK, Tilanus HW, van Lanschot JJ, et al: The influence of preoperative weight loss on the postoperative course after esophageal cancer resection. J Thorac Cardiovasc Surg 2014;147:490-495.

4 Correia MI, Caiaffa WT, da Silva AL, et al: Risk factors for malnutrition in patients undergoing gastroenterological and hernia surgery: an analysis of 374 patients. Nutr Hosp 2001;16:59-64.

5 Marin FA, Lamônica-Garcia VC, Henry MA, et al: Grade of esophageal cancer and nutritional status impact on postsurgery outcomes. Arq Gastroenterol 2010;47:348-353.

6 Braga M, Ljungqvist O, Soeters P, et al: ESPEN Guidelines on Parenteral Nutrition: surgery. Clin Nutr 2009;28: 378-386.

7 Johansen N, Kondrup J, Plum LM, et al: Effect of nutritional support on clinical outcome in patients at nutritional risk. Clin Nutr 2004;23:539-550.

8 Jie B, Jiang ZM, Nolan MT, et al: Impact of nutritional support on clinical outcome in patients at nutritional risk: a multicenter, prospective cohort study in Baltimore and Beijing teaching hospitals. Nutrition 2010;26:10881093.

9 Wang JY, Hong X, Chen GH, et al: Clinical application of the fast track surgery model based on preoperative nutritional risk screening in patients with esophageal cancer. Asia Pac J Clin Nutr 2015;24:206-211.

10 Braga M, Wischmeyer PE, Drover J, et al: Clinical evidence for pharmaconutrition in major elective surgery. JPEN J Parenter Enteral Nutr 2013;37(5 suppl):66S-72S.

11 Cerantola Y, Hübner M, Grass F, et al: Immunonutrition in gastrointestinal surgery. Br J Surg 2011;98:37-48.

12 Marimuthu K, Varadhan KK, Liungqvist O, et al: A meta-analysis of the effect of combinations of immune modulating nutrients on outcome in patients undergoing major open gastrointestinal surgery. Ann Surg 2012;255: 1060-1068. 
13 Zhang Y, Gu Y, Guo T, et al: Perioperative immunonutrition for gastrointestinal cancer: a systematic review of randomized controlled trials. Surg Oncol 2012;21:e87-e95.

14 Weimann A, Braga M, Harsanyi L, et al: ESPEN Guidelines on Enteral Nutrition: surgery including Organ Transplantation. Clin Nutr 2006;25:224-244.

15 Wong CS, Aly EH: The effects of enteral immunonutrition in upper gastrointestinal surgery: a systematic review and meta-analysis. Int J Surg 2016:29:137-150.

16 Sultan J, Griffin SM, Di Franco F, et al: Randomized clinical trial of omega-3 fatty acid-supplemented enteral nutrition versus standard enteral nutrition in patients undergoing oesophagogastric cancer surgery. Br J Surg 2012;99:346-355.

17 Hallay J, Kovács G, Kiss S, et al: Changes in the nutritional state and immune-serological parameters of esophagectomized patients fed jejunaly with glutamine-poor and glutamine-rich nutriments. Hepatogastroenterology 2002;49:1555-1559.

18 Sakurai Y, Masui T, Yoshida I, et al: Randomized clinical trial of the effects of preoperative use of immuneenhancing enteral formula on metabolic and immunological status in patients undergoing esophagectomy. World J Surg 2007;31:2150-2157.

19 Takeuchi H, Ikeuchi S, Kawaguchi Y, et al: Clinical significance of perioperative immunonutrition for patients with esophageal cancer. World J Surg 2007;31:2160-2167.

20 Aiko S, Yoshizumi Y, Ishizuka T, et al: Enteral immune-enhanced diets with arginine are safe and beneficial for patients early after esophageal cancer surgery. Dis Esophagus 2008;21:619-627.

21 Fukuda T, Seto K, Yamada K, et al: Can immune-enhancing nutrients reduce postoperative complications in patients undergoing esophageal surgery? Dis Esophagus 2008;21:708-711.

22 Ryan AM, Reynolds JV, Healy L, et al: Enteral nutrition enriched with eicosapentaenoic acid (EPA) preserves lean body mass following esophageal cancer surgery: results of a double-blinded randomized controlled trial. Ann Surg 2009;249:355-363.

23 Aiko S, Kumano I, Yamanaka N, et al: Effects of an immune-enhanced diet containing antioxidants in esophageal cancer surgery following neoadjuvant therapy. Dis Esophagus 2012;25:137-145.

24 Nagano T, Fujita H, Tanaka T, et al: A randomized controlled trial comparing antioxidant-enriched enteral nutrition with immune-enhancing enteral nutrition after esophagectomy for cancer: a pilot study. Surg Today 2013;43:1240-1249.

25 Kubota K, Kuroda J, Yoshida M, et al: Preoperative oral supplementation support in patients with esophageal cancer. J Nutr Health Aging 2014;18:437-440.

26 Matsuda Y, Habu D, Lee S, et al: Enteral diet enriched with $\omega$-3 fatty acid improves oxygenation after thoracic esophagectomy for cancer: a randomized controlled trial. World J Surg 2017;41:1584-1594.

27 Kitagawa H, Namikawa T, Yatabe T, Munekage M, Yamasaki F, Kobayashi M, Hanazaki K: Effects of a preoperative immune-modulating diet in patients with esophageal cancer: a prospective parallel group randomized study. Langenbecks Arch Surg 2017;402:531-538.

28 Healy LA, Ryan A, Doyle SL, et al: Does prolonged enteral feeding with supplemental omega-3 fatty acids impact on recovery post-esophagectomy: results of a randomized double-blind trial. Ann Surg 2017;266: 720-728.

29 Zhou M, Martindale RG: Arginine in the critical care setting. J Nutr 2007;137(6 suppl 2):1687S-1692S.

30 Mudge L, Isenring E, Jamieson GG: Immunonutrition in patients undergoing esophageal cancer resection. Dis Esophagus 2011;24:160-165.

31 Bertolini G, Lapichino G, Radrizzani D, et al: Early enteral immunonutrition in patients with severe sepsis: results of an interim analysis of a randomized multicenter clinical trial. Intensive Care Med 2003;29:834-840.

32 Dent DL, Heyland DK, Levy H, et al: Immunonutrition may increase mortality in critically ill patients with pneumonia: results of a randomized trial. Crit Care Med 2003;30:A17.

33 McClave SA, Martindale RG, Vanek VW, et al: Guidelines for the provision and assessment of nutrition support therapy in the adult critically ill patient: Society of Critical Care Medicine (SCCM) and American Society for Parenteral and Enteral Nutrition (A.S.P.E.N.). JPEN J Parenter Enteral Nutr 2009;33:277-316.

34 Yoshida S, Matsui M, Shirouzu Y, et al: Effects of glutamine supplements and radiochemotherapy on systemic immune and gut barrier function in patients with advanced esophageal cancer. Ann Surg 1988;227:485-491.

35 Sunpaweravong S, Puttawibul P, Ruangsin S, et al: Randomized study of antiinflammatory and immune-modulatory effects of enteral immunonutrition during concurrent chemoradiotherapy for esophageal cancer. Nutr Cancer 2014;66:1-5.

36 Kubota H, Matsumoto H, Higashida M, et al: Eicosapentaenoic acid modifies cytokine activity and inhibits cell proliferation in an oesophageal cancer cell line. Anticancer Res 2013;33:4319-4324.

37 Mizoguchi K, Ishiguro H, Kimura M, et al: Induction of apoptosis by eicosapentaenoic acid in esophageal squamous cell carcinoma. Anticancer Res 2014;34:7145-7150.

38 Chen H, Ward MH, Graubard BI, et al: Dietary patterns and adenocarcinoma of the esophagus and distal stomach. Am J Clin Nutr 2002;75:137-144.

39 Wilson KT, Fu S, Ramanujam KS, et al: Increased expression of inducible nitric oxide synthase and cyclooxygenase-2 in Barrett's esophagus and associated adenocarcinomas. Cancer Res 1998;58:2929-2934.

40 Mehta SP, Boddy AP, Cook J, et al: Effect of n-3 polyunsaturated fatty acids on Barrett's epithelium in the human lower esophagus. Am J Clin Nutr 2008;87:949-956. 
41 Osland E, Hossain MB, Khan S, et al: Effect of timing of pharmaconutrition (immunonutrition) administration on outcomes of elective surgery for gastrointestinal malignancies: a systematic review and meta-analysis. JPEN J Parenter Enteral Nutr 2014;38:53-69.

42 Fujitani K, Tsujinaka T, Fujita J, et al: Prospective randomized trial of preoperative enteral immunonutrition followed by elective total gastrectomy for gastric cancer. Br J Surg 2012;99:621-629.

43 Serhan CN, Clish CB, Brannon J, et al: Novel functional sets of lipid-derived mediators with antiinflammatory actions generated from omega-3 fatty acids via cyclooxygenase 2-nonsteroidal antiinflammatory drugs and transcellular processing. J Exp Med 2000;192:1197-1204.

44 Moro K, Nagahashi M, Ramanathan R, et al: Resolvins and omega three polyunsaturated fatty acids: clinical implications in inflammatory diseases and cancer. World J Clin Cases 2016;4:155-164.

45 Uno H, Furukawa K, Suzuki D, et al: Immunonutrition suppresses acute inflammatory responses through modulation of resolvin E1 in patients undergoing major hepatobiliary resection. Surgery 2016;160:228-236. 